\title{
Believe It or Not: Factors Influencing Credibility on the Web
}

\author{
C. Nadine Wathen \\ Faculty of Information and Media Studies, The University of Western Ontario, London, Ontario, \\ Canada N6A 5B7. E-mail: cwathen@uwo.ca \\ Jacquelyn Burkell \\ Faculty of Information and Media Studies, The University of Western Ontario, London, Ontario, \\ Canada N6A 5B7
}

\begin{abstract}
This article reviews selected literature related to the credibility of information, including (1) the general markers of credibility, and how different source, message and receiver characteristics affect people's perceptions of information; (2) the impact of information medium on the assessment of credibility; and (3) the assessment of credibility in the context of information presented on the Internet. The objective of the literature review is to synthesize the current state of knowledge in this area, develop new ways to think about how people interact with information presented via the Internet, and suggest next steps for research and practical applications. The review examines empirical evidence, key reviews, and descriptive material related to credibility in general, and in terms of on-line media. A general discussion of credibility and persuasion and a description of recent work on the credibility and persuasiveness of computer-based applications is presented. Finally, the article synthesizes what we have learned from various fields, and proposes a model as a framework for much-needed future research in this area.
\end{abstract}

\section{Introduction}

People seek information for comfort, support, empowerment, the impetus and knowledge to act, or simply to learn. Information is normally seen as that which has meaning, in that it reduces uncertainty for the seeker (Belkin, 1978; Buckland, 1991; Dervin, 1977); information can also, however, increase uncertainly and create more dissonance (Rogers, 1995). The information-seeking process is iterative, and depends largely on the seeker's specific situation, along with the broader context (Dervin, 1992, 1997). The impact of information can be limited to learning: information can simply be stored and recalled. In many cases, however, there is a much larger impact. Information can be passed on

(C) 2002 Wiley Periodicals, Inc.

DOI: $10.1002 /$ asi.10016 to others, it can be used to make decisions, and it can affect attitudes and behaviors. We encounter a great deal of information in our everyday lives, most of which we filter out, retaining only that which is useful to us. One of the criteria we use to filter information is its credibility, or believability.

Although users of information face the issue of filtering out information, those who produce information have a different problem. Information producers would like theirs to be the message that is attended to, recalled, or acted upon. If a user rejects new information as not credible, that information will not be learned, nor can it have any other impact. Thus, the provision of credible information serves as a necessary, but not sufficient, component of any process designed to influence knowledge, attitudes or behavior.

In the psychological literature, a great deal of research has examined the issues of attitude formation and behavior change. In the mid-1980s, Petty and Cacioppo (1986) provided a useful way for thinking about how people become persuaded-the Elaboration Likelihood Model (ELM). In their model, persuasive communications that invoke careful, cognitive evaluation lead to the most persistent behavior and attitude changes. This "central route" to persuasion, however, requires considerable effort, and is not possible for all persuasive messages encountered. By contrast, attitudes formed by less cognitively demanding means, such as classical conditioning, inferences, or heuristics-so-called "peripheral routes"- are less persistent, stable, and predictive of behaviour (Petty, Cacioppo, Sekides, \& Strathman, 1988). A key early stage in the persuasion process is the receiver's judgment of the credibility of the information. As such, credibility is an "extramessage cue" that is a critical aspect of the persuasiveness of a message (Petty \& Cacioppo, 1986).

Given that credibility strongly influences the impact of a message, it becomes important to understand how users decide what to believe. The question of what marks credible 
information has been studied within various academic disciplines, including information science, psychology, sociology, marketing, communications, and health sciences. Message credibility is generally agreed to result from an interaction of source characteristics (e.g., expertise, trustworthiness), message characteristics (related to message content, encompassing factors such as plausibility, internal consistency, and quality), and receiver characteristics (e.g., cultural background, previous beliefs). This notion arises in even the earliest discussions of credibility. In an important review of credibility research and theory, Self presents a summary of early Socratic and Aristotelian ideas about credibility: "First, sources are credible because their message's rightness is perceived by the audience. Second, sources are credible because they rightly read how to reveal themselves to particular audiences. And, third, sources are perceived to be credible because of audience characteristics" (Self, 1996, p. 423).

Research on source credibility examines the impact of personal (or organizational) characteristics such as expertise or trustworthiness on the "believability" of the message that is delivered. Other bodies of literature examine the impact of message and receiver on credibility, while still other research investigates the interaction of these three factors on credibility assessment. The impact of delivery medium (which can be construed as an aspect of message source) on credibility assessment has not been explored to the same degree. Research that does examine media effects on credibility tends simply to compare the credibility of messages in different media (Johnson \& Kaye, 1998) without exploring how these credibility differences arise. Thus, there are little data on questions such as the impact of personalized messages versus broadcast delivery, or the impact of channel fidelity (high vs. low) on credibility assessment. One important question is whether new media introduce new factors into credibility assessment. For example, as we move from face to face to television as a communication medium, does image size become important in the determination of message credibility?

The advent of the Internet as a new and widely used method for the delivery of information raises the question of how credibility is assessed in this medium. The number of Internet users is large, and ever-increasing: in 2000, the number of North American Internet users exceeded 100 million (e-land, 2000). A well-documented concern of both information providers and information seekers on the Internet is the decline in the perceived quality of Internet information, or at least the growing belief that high-quality information will be impossible to find amidst the vast amount of lower quality, unfiltered information (Eysenbach, 2000; Shon \& Musen, 1999). Thus, both information providers and users of the Internet are interested in the question of how users can and will assess the credibility of information presented through that medium.

This literature review attempts, based on previous research, to provide some insight into this question. We begin by providing a definition of credibility. We then discuss, in turn, the factors that influence credibility in traditional media (including personal interaction) and electronic information (including information on the Web). The specific case of Internet health information is then examined, followed by a discussion of the evidence that is reviewed in the article and general recommendations to help information producers ensure that their Internet information is seen as credible by audiences.

Finally, a proposed model for how users judge the credibility of on-line information is presented. This model is in part an attempt to synthesize existing evidence and in part a call for empirical research to examine the key gaps in the literature outlined in the review.

\section{A Definition of Credibility}

What do we mean by "credibility"? At its simplest, credibility can be defined as "believability" (Fogg, 1999; Tseng \& Fogg, 1999). Credible sources are described as "trustworthy" and having "expertise" (Self, 1996). Sources are judged as credible based on perceived competence, character, composure, dynamism and sociability (Burgoon, Bonito, Bengtsson, Cederberg, Lundeberg, \& Allspach, 2000). Tseng and Fogg (1999) identify four types of source credibility. Presumed credibility arises from the assumptions of the perceiver. For example, stereotypes about an object or source, such as "car salesmen are generally dishonest," can lead to a credibility judgment, such as "don't believe what the car salesman says," absent any other cues. Reputed credibility is based on source labels. Those sources labeled "Doctor" or "Professor," for example, are perceived as credible by virtue of the label. Surface credibility is assigned based on a user's simple inspection of superficial characteristics - i.e., "judging a book by its cover." Finally, experienced credibility is based on a user's first-hand experience with a source over time. This is the most complex, and reliable, method of making credibility judgments (Fogg, 1999; Tseng \& Fogg, 1999).

Research on credibility operationalizes the concept in one of three ways. First, credibility can be assessed directly by asking respondents to indicate whether information and/or information sources are believable. This direct assessment, however, is not always used. Instead, credibility of information is often inferred on the basis of proxy measures. These proxy measures include: (1) knowledge change [on the relatively weak assumption that only credible information is processed enough to be recalled (e.g., Self, 1996)]; and (2) attitude or behavior change [on the stronger assumption that information must be credible, and thus believed, before it can affect attitudes or behavior (e.g., Petty et al., 1988)].

\section{Credibility in Traditional Media}

Message familiarity can influence judged credibility, with more familiar messages being judged as more credible (Self, 1996). In some cases, receiver and source character- 
TABLE 1. Examples of factors influencing credibility (print and interpersonal media).

\begin{tabular}{|c|c|c|c|c|}
\hline Source & Receiver & Message & Medium & Context \\
\hline - Expertise/Knowledge & - Issue relevance & - Topic/content & - Organization & - Distraction/“noise” \\
\hline - Trustworthiness & $\begin{array}{l}\text { - Motivation (i.e., need for } \\
\text { the information) }\end{array}$ & - Internal validity/consistency & - Usability & $\begin{array}{l}\text { - Time since message } \\
\text { encountered }\end{array}$ \\
\hline - Credentials & $\begin{array}{l}\text { - Prior knowledge of the } \\
\text { issue }\end{array}$ & - Plausibility of arguments & - Presentation & \\
\hline - Attractiveness & - Issue involvement & $\begin{array}{l}\text { - Supported by data or } \\
\text { examples }\end{array}$ & - Vividness & \\
\hline $\begin{array}{l}\text { - Similarity to receiver beliefs/ } \\
\text { context }\end{array}$ & - Values/beliefs/situation & - Framing (loss or gain) & & \\
\hline \multirow[t]{2}{*}{ - Likeability/Goodwill/Dynamism } & $\begin{array}{l}\text { - Stereotypes about source or } \\
\text { topic }\end{array}$ & - Repetition/familiarity & & \\
\hline & - "Social location" & - Ordering & & \\
\hline
\end{tabular}

istics interact in credibility judgments. Message sources chosen to match audience attitudes and context lead to higher perceived credibility and better recall of the information. Indeed, health behaviors in a group that received a tailored message from a credible (and familiar) source were changed to comply with the message (Campbell et al., 1999). Early studies by Hovland and colleagues (1949, 1953, cited in Self, 1996, showed that messages from both high- and low-credibility sources (those with more or less "trustworthiness" and "expertise") were learned equally well. In assessment immediately following information presentation, messages from high-credibility sources changed attitudes more than those from low credibility sources. In a follow-up 4 months after the initial delivery of the message, however, subjects could no longer remember the source of the message, and messages from high- and low-credibility sources assumed similar levels of persuasiveness. It may be, therefore, that the primary influence of credibility factors is at the point of "first contact" between the receiver and the message: the means by which the message is made persuasive might not matter in the long run, only that it was persuasive in the first place.

Wilson and Sherrell (1993) performed a meta-analysis of empirical studies that measured factors influencing credibility perceptions. Studies that qualified for the analysis measured three types of effects: (1) those arising from manipulation of source characteristics (e.g. expertise, attractiveness, similarity to user); (2) those due to the message (e.g. the issue being communicated); and (3) those due to the medium or channel. Their operational definition of credibility involved internalization of the message: "a communication source possesses credibility if his/her statements are considered truthful and valid ... and thus worthy of serious consideration. A member of a target audience may accept (internalize) the influence of the communication source because the advocated behavior is congruent with his/her own value system." (p. 102). The results of the meta-analysis indicate that (1) source effects overall have a relatively small effect on knowledge/attitude/behavior change or persuasion ( $9 \%)$; (2) when source characteristics are involved, expertise is the most important one; and (3) the source by receiver involvement interaction is significant, and consistent with the predictions of the ELM (i.e., that highly involved/motivated users take a central route, relying less on peripheral cues and more on the content of the message) (Wilson \& Sherrell, 1993).

Thus, the majority (but not all) of the available evidence conceptualizes credibility as a multidimensional construct whose two main source-related components are trustworthiness and expertise, along with dynamism, likeability, and goodwill, among others (see also O'Hara, Netemyer, \& Burton, 1991). In the determination of credibility, source effects interact with receiver-related factors, such as level of involvement and attitudes (Petty \& Cacioppo, 1990). If the audience is less involved with the topic, source characteristics will have more influence on the construct under investigation (e.g., knowledge/attitudes/behaviors). Source characteristics also interact with message-related factors, such as discrepancy (from previous beliefs), incongruity in content, or timing of source identification within the message. For example, if a message is high in discrepancy, low in incongruity, and if the source is identified early in the message, then high-credibility sources will tend to have a greater impact on knowledge/attitudes/behaviors than low credibility sources (Wilson \& Sherrell, 1993).

Slater and Rouner (1996) postulate that credibility assessments are based on (1) knowledge and attitudes about the source specifically; (2) attitudes about credentials (i.e., inferred or reputed credibility); and (3) quality of the message, including its presentation, plausibility, and whether it is supported by data or good examples. The third set of factors, they claim, is not well studied. This research showed that the way a message is presented (e.g. wellwritten, -produced, -organized) can influence how the source is perceived. Source credibility ratings made before message presentation can be mediated by message characteristics: well-presented messages lend credibility to the source, while poorly presented messages detract from cred- 
ibility. However, if a source is initially seen as expert and credible, the mediating effect is not as strong: the message and source are more likely to retain credibility in the face of poor presentation (Slater \& Rouner, 1996).

Table 1 summarizes some of the variables related to source, message, receiver, context, and media characteristics discussed above in the context of traditional (mainly print and interpersonal) media. All of these factors are relevant in the on-line environment, though the interpretation or application of them may differ.

\section{Credibility in Electronic Media}

Several authors in information science have developed ways to think about electronically delivered information. They identify how this type of information differs, at a cognitive, information processing level, from more traditional forms. Olaisen (1990) discusses factors related to information quality and how "cognitive authority" is established for electronic information. He equates cognitive authority to influence, and influence to credibility. He states what is generally known in information science theorythat although personal information sources may be the most trusted, they are not necessarily the most expert: we turn to other sources when expertise or competence is required. He states: "when we are processing information we will give credit and authority to certain persons and sources depending on our social location. One's social location ... will greatly influence quality factors like credibility (i.e., reliability), relevance and perceived value of information" ( $p$. 92). He goes on to speculate whether the electronic transfer of information has effectively changed our "social location" (our location in time and space and in a network of social relationships) and the types of social networks we inhabit (Olaisen, 1990). For texts, cognitive authority (i.e., credibility) ratings are made based on the perceived expertise of the author and/or publisher, or referral to the text from a credible source. Olaisen asks: "[given that] we can store, organize and distribute electronic information in a completely different way than we organized printed information ... do we then give a different credibility or cognitive authority to electronic information ...?" (p. 92).

Olaisen (1990) among others (Fogg, 1999; Petty \& Cacioppo, 1986), differentiates between factors related to sources/messages/receivers and those related to design features of the medium. Calling the former "cognitive" qualities, and the latter "technical" qualities, he ranks various factors from each in terms of importance to cognitive authority perceptions. For "cognitive" factors, influence, trustworthiness, competence, reliability, and relevance determine cognitive authority. On the "technical" side, form, novelty, accessibility, and flexibility are key. Combined, cognitive and technical features make up what he terms "institutional quality" (Olaisen, 1990)—presumably, the greater the "institutional quality," the higher the judgment of cognitive authority, and thus the more credible and persuasive the information.
Interestingly, Olaisen goes on to say that cognitive authority tends to be attributed more to informal than formal sources of information. Intervening variables, such as time and extrinsic value (e.g., price) can also influence cognitive authority (the nearer in time or the more expensive the item, the more cognitive authority will be granted).

It has been proposed that the Internet combines the broad reach advantages of mass communication channels with the persuasion characteristics of interpersonal channels by allowing for give and take between the message source and receiver. The Internet, therefore, may have a greater ability than other mass media to make use of principles of consumer behavior to enhance information provision and uptake (Cassell, Jackson, \& Cheuvront, 1998).

In a direct comparison of "new" versus "old" media, Johnson and Kaye examined Web users' perceptions of the credibility of Web-delivered information compared to that delivered more traditionally. The study compared political information in paper-based newspapers, magazines, candidate literature, or issue-oriented sources, to similar information sources on-line. The sample $(N=308)$ was a selfselected group (by virtue of choosing to complete the online survey) of regular consumers of political information who described themselves as heavy Internet users $(>13$ hours/week). Users found on-line newspapers and candidate literature to be significantly more credible than their traditional counterparts. Magazines and issue-oriented material were found to be equally as credible whether delivered traditionally or on-line. Of interest, users found political issue-oriented Web sites more credible than either on-line newspapers or magazines. Candidate literature was not deemed credible. However, in terms of overall credibility of political information, no source, either delivered on-line or traditionally, was rated more than "somewhat" credible-a statement more, perhaps, about the subject matter than the medium, or more indicative of the public's general and growing skepticism about mass media (Johnson \& Kaye, 1998). Of relevance to the topic at hand is that the medium, at least for political information, did not influence credibility as much as the source of the information. Thus, when source and message are separated from medium, as is often not the case in the current literature relevant to this topic, there are factors that distinguish between the three, and influence credibility.

Rieh and Belkin (1998, 2000), in two qualitative studies, examined cognitive authority and quality ratings used by scholars for information presented on the Web. They postulated that these judgments serve as filtering mechanisms, allowing people to more efficiently manage the amount of information they have to process. This is especially important in the Web environment, because the amount of information available is virtually limitless (Rieh \& Belkin, 1998). According to these authors, the quality checks used by scholars for print materials_-presence of peer review, refereeing, publisher reputation-are more difficult to apply in the Web environment. In addition, direct experience with the source is less likely to be available to users, given the 
newness of the medium, and the often-ambiguous nature of the source.

Rieh and Belkin (1998) found that the types of information needs that lead scholars to the Web (i.e., to learn about a new topic/get started in an unfamiliar area) do not differ from those triggering searches of traditional media. Quality judgments of Web information are also based on the perceived credibility of the source. Users in the Rieh and Belkin $(1998,2000)$ studies sought source credibility markers to replace those used for print media, identifying aspects such as suffixes to the URL address (i.e., .edu, .org, or .gov). These, denoting educational, nonprofit, and government sources, respectively, were rated as more credible than were commercial sites (.com). At the individual source level, users looked for reputed or referred credibility markers, such as a statement of the author's credentials and institutional affiliations. These were seen as particularly important due to the lack of publication controls on the Web. Related to this is the use of referrals to specific sites from trusted sources (a colleague, known expert or journal article). These referrals were often cited as reasons for visiting a given site. Of interest was that, even when seemingly believable information was found on the Web using the credibility and quality checks above, the scholars serving as subjects in the first study still felt the need to verify the information by referring back to a traditional expert source.

The authors conclude that there are seven criteria that affect credibility of Web information: source, content, format, presentation, currency, accuracy, and speed of loading: "since authority is not necessarily identified in the Web environment, [subjects] used many different, and new characteristics of Web information objects (pages) in order to make their judgments of authority and credibility" ( p. 288).

Fogg and colleagues (Fogg et al., 2000) conducted a large-scale $(N=1,410)$ on-line survey of Web users in the United States and Finland to examine the elements that user's report as affecting their judgments of Web site credibility. They found seven factors that influence credibility: "real-world feel"; ease of use, expertise, trustworthiness, and message tailoring had positive effects on credibility ratings. Commercial implications and amateurism negatively affect credibility. These authors conclude that people use the same types of criteria for assessing on-line information as they use for traditional media-indeed they seek out familiarity in this new environment (Fogg et al., 2000).

Information on the Internet may benefit from a sort of presumed credibility. Many users assume that if someone expends the effort and financial resources to provide information on a Web site, then the quality of that information must be at least adequate (Feightner, Quintana, Marshall, \& Wathen, 1999). In addition, surface credibility is evident in the assessment of Internet information. Information coupled with a well-designed interface and attractive graphics may result, in the absence of more substantive cues, in a tendency for users to make a positive credibility judgment (Critchfield, 1998). Some surface characteristics, such as errors unrelated to content, negatively influence credibility.
Tseng and Fogg (1999) conclude that "small errors by computers have disproportionately large effects on perceptions of credibility" (p. 43). Links to and from other Web sites may act as referrals, implying third party endorsement, which increases the reputed credibility of the site (Fogg \& Tseng, 1999). First-hand experience with a Web site also informs credibility judgments (Reih \& Belkin, 2000), indicating that experiential credibility is important in assessing Web-based information.

There is some evidence of an interesting interaction between user characteristics and errors in an electronic information interface. User expertise tends to influence how computing devices and electronic information are perceived: a more technologically sophisticated user will tend to scrutinize the source or object much more closely and more critically, leading to greater skepticism about the object and the tendency to assign low credibility (Tseng \& Fogg, 1999; note, however, that there are some mixed results in this area, reviewed in Tseng \& Fogg, 1999). In evaluation of Web-based information, there also appears to be a utilitarian aspect to credibility assessment. User need for information is correlated to credibility judgments- the greater the need, the more likely the user is to accept the information as credible (Tseng \& Fogg, 1999). These characteristics interact with two types of evaluation errors: (1) gullibility errors, defined as "blind faith" in a technology or source; and (2) incredulity errors, defined as "blind skepticism" in the source. Simply speaking, novices to both the topic and the computing process and those with a greater need for information will tend to make gullibility errors, while expert users will tend to incredulity. Fogg and Tseng point out that most educational and research efforts are directed at decreasing gullibility errors by educating novices. However, the goal should be to find the right balance of knowledge, experience, and needs to decrease gullibility errors without increasing incredulity errors-i.e., to design systems that match the needs of users and instill confidence in the information being provided.

\section{Internet Health Information}

An estimated $43 \%$ of Internet users go on-line to gather health information on over 34,000 health related Web sites (DMOZ Open Directory Project, 2000). There are numerous advantages to using the Internet to seek and provide health information. Consumers can access information in the privacy of their own home and at convenient times. Furthermore, the information can be tailored to the specific needs and desires of the consumer, based on such factors as client demographics (e.g., a user can specify the language in which the information is provided) and learning style (e.g., a choice between graphical or written forms of the same information). In addition, the Internet presents the opportunity to support written material with multimedia features, and provides a level of interactivity not available in other formats. The Science Panel on Interactive Communication and Health (SciPICH) identified the following advantages 
of interactive health communication (Robinson, Patrick, Eng, \& Gustafson, 1998): (a) improved opportunity to tailor messages; (b) improved use of media to match learning styles of users; (c) increased possibility for users to remain anonymous, which may increase their willingness to engage in frank discussions; (d) increased access to information and support on demand; (e) increased opportunity for interaction with health professionals and/or support from others; (f) enhanced dissemination capabilities and opportunity to keep content current.

A major disadvantage of the Internet, arising from the ease of publication and distribution in this medium, is the potential for the distribution of inaccurate medical information from unqualified sources (Jadad \& Gagliardi, 1998; Jadad, Hayes, Hunt, \& Browman, 2000; Richards et al., 1998; Robinson et al., 1998; Winker, Flanagin, Chi-Lum, White, Andres, Kennett, DeAngelis, \& Musacchio, 2000). In an assessment of 60 articles published on the Internet by traditional medical sources, McClung, Murray, and Heitlinger (1998) found that only $20 \%$ followed established guidelines for the treatment of childhood diarrhea. Similarly, Impicciatore and colleagues found that only 4 of 41 articles on the World Wide Web closely followed guidelines for managing fever in children at home (Impicciatore, Pandolfini, Casella, \& Bonati, 1997). SciPICH suggests that misleading information may lead to inappropriate treatment or delays in seeking necessary medical care (Robinson et al., 1998).

Given the widespread use of the Internet for health information along with the critical nature of the information that users seek, it can be argued that the use of health information presents a great need for credibility assessment (Jadad \& Gagliardi, 1998; Jadad et al., 2000; Winker et al., 2000).

\section{A Potential Solution: Credibility Rating Systems}

Consumers of health information identify credibility as an important aspect of useful information. Bunn (1993) used a multidimensional scaling approach to identify sources of health information and the important characteristics of those sources. In order of use, the sources were: doctors; mass media; other medical professionals; health publications; disease foundations/associations; libraries; and prerecorded messages. Source characteristics perceived as most important, in order, were: current/up-to-date; accurate; easy-to-understand; credible; easy-to-get; exactlywhat-I-want; and confidential. Doctors were the most credible and accurate sources of medical information, but ranked lowest in "easy-to-understand" and "easy-to-get." Mass media were easiest to access and understand, but lowest in credibility, accuracy and "exactly-what-I-want" (Bunn, 1993).

In a recent study of on-line health information needs, Quintana and colleagues explored, in focus groups with health care consumers, issues related to the provision of preventive health information on the Internet. The impor- tance of information credibility and trust in the information source was a main finding. Consumer advocacy groups, such as the Cancer Society and the Lung Association, were seen as very credible, and were trusted by participants. Well-known organizations such as the Mayo Clinic and governments were also identified as credible sources, though some participants were more skeptical about information from governments. Generally, participants did not speak favorably about corporate Web sites, especially those of drug companies. Collectively, participants identified a number of characteristics that would increase the credibility of a Web site: (1) the provider organization is known to them (experiential credibility); (2) the Web site was publicized in their doctor's office (reputed credibility); (3) the Web site identified the sources of information, including references to medical evidence; and (4) the Web site did not include corporate advertising (Feightner et al., 1999; Quintana, Feightner, Wathen, Sangster, \& Marshall, 2001).

More often than not, the Internet presents medical information in a conversational fashion rather than as a tool for effective health care communication and decision-making. Several authors (Eysenbach, 2000; Eysenbach \& Diepgen, 1998; Impicciatore et al., 1997; Silberg, Lundberg, \& Musacchio, 1997) call for the institution of core standards, allowing consumers and professionals alike to reasonably judge the credibility and reliability of Internet health information and to make informed decisions about how to apply the information in the real world.

One approach to achieving these goals is to develop rating instruments to assess the quality of Internet health information. In effect, ratings serve as a form of "expertise marker," conveying what Tseng and Fogg (1999) term "reputed credibility" to the Web site. In a review of published criteria for evaluating health related Web sites, the most frequently cited criteria included: content (quality, reliability, accuracy, scope, and depth); design and esthetics of site, disclosure of authors, sponsors, developers, currency of information (frequency of update, maintenance of site), authority of source, ease of use, and accessibility and availability (Lim, Eng, Deering, \& Maxfield, 1999). Four basic criteria, consistent with credibility markers, are common to existing quality ratings scales: authorship, attribution, disclosure and currency (Shon \& Musen, 1999). In the on-line context, these publishing criteria serve as a proxy for information quality, and in turn, for credibility markers.

Jadad and Gagliardi (1998), however, raise concerns about these rating systems. They concluded that it was unclear "whether [instruments to evaluate health information on the Internet] should exist in the first place, whether they measure what they claim to measure, or whether they lead to more good than harm" (p. 614). Furthermore, these authors questioned whether users would even notice evaluations of the information. More recent initiatives, such as the MedPICS project (Eysenbach, 2000; Eysenbach \& Diepgen, 1998) attempt to overcome these criticisms.

Although these early efforts at standardization and quality control can provide users and Web site developers with 
some basic guidelines, there is still much research to be done to understand what quality control measures users are looking for (if any), how to apply these is a consistent and meaningful fashion, and how to inform users of Internet information, including health information, that these tools and measures exist. As a first step, the identification of key Internet information credibility markers could aid the development of usable quality rating instruments. Clearly, this is still an evolving field, but gains made in health-related Internet research will be generally applicable to Web-delivered information.

\section{Discussion}

It is evident from the literature cited above that credibility is complex and multifaceted. Source, receiver, and message characteristics all interact in the assessment of information credibility. Given that the message itself and the audience to whom it is targeted are largely fixed, it is clear that information providers should pay attention to aspects of the source of the information if they want to ensure that users see their information as credible and therefore useful. It is also evident that the medium of delivery has an impact on credibility assessment.

Obviously, the message itself is critical for information credibility. The message should be internally consistent, and clearly presented. Audience characteristics are also important: audiences that are already receptive to a message will be more likely to view the information as credible.

With regard to source effects, face-to-face interaction is the most basic form of information provision. Research examining credibility in this type of interaction has repeatedly demonstrated that the individual or organization that is the source of the information has a great influence on the assessed credibility. Expertise, knowledge, and trustworthiness are qualities that mark credible sources of information. Users react directly to the qualities of the information source; they also utilize assumptions (e.g., stereotypes about group membership), reputation (assessments of others regarding the source) and labels (e.g., "Dr.," "Professor") in their assessment of credibility. Matching the source to the audience is also important: different audiences trust different sources of information (Campbell et al., 1999).

When an intermediary medium is used to transmit information, different aspects come into play in the assessment of credibility, as the medium itself has some influence. Surface aspects of the presentation (presence of errors, pleasantness of graphics, etc.) have an impact on assessed credibility. In addition, audience attitudes toward the medium itself may influence judgment of credibility of information presented in that medium; in the Johnson and Kaye (1998) study, Web users rated the same information on the Web as more credible than information in other forms such as newspapers.

Information on the Internet is delivered by machine, not by a person or organization. As such, it might be assumed that source characteristics that affect credibility judgement in face-to-face interactions are irrelevant to credibility assessment in this new medium. There is, however, a large body of research demonstrating that people treat computers exactly as they would other people in many social interactions (Nass \& Moon, 2000). The most rudimentary cues serve to engage these social mechanisms: one computer will be perceived as more "friendly" than another if it is programmed simply to address you by name after you have provided the information. As electronic interfaces become more sophisticated, the social aspects of human-computer interactions will become increasingly powerful. There is every reason to believe, therefore, that electronic information sources will engender reactions much like human sources of information, with the same qualities marking credible sources.

Surface aspects of the presentation are also relevant to credibility assessment. For traditional media, these might include physical appearance or attractiveness of a speaker, glossiness of a magazine or quality of the paper used in a book. For on-line media, these include the appearance of the site's homepage (colors, graphics, etc.), the usability of the interface, how well the information is organized-in general, how well the site is designed. For Internet information, even a single spelling mistake can give the impression of "amateurism" and lead the user to reject the site as not credible. Thus, the computing object itself comes under scrutiny in the assessment of credibility, and it is likely that the general attitude of the user toward computers will have an impact on the assessed credibility of information presented on the Internet.

Table 2 summarizes the credibility-related variables that have been identified specific to computer-based media. It is interesting to note that within this (albeit new and limited) literature, the source and medium are usually treated as essentially the same construct. Many authors also include message characteristics in this category. In addition, the focus of study and applied design efforts is clearly on the source/medium/message, rather than the receiver or context.

Emerging from the literature described above is the notion that users are judging many different aspects of information as they assess credibility. These include the message itself, their reaction to the message, the source of the message, and the medium in which the message is presented. Although these factors appear to be consistent across all media, there are differences in the assessment of credibility in different contexts. In particular, for electronic information (including information presented on the Internet), surface aspects of the presentation take on particular significance, and the general attitude of the user toward computers has an impact on credibility.

An important question is how these different aspects of credibility assessment interact. Based on extrapolation of the Petty and Cacioppo Elaboration Likelihood Model (ELM) to computing technologies, Fogg and Tseng (1999) propose three possible models of credibility evaluation, ranging from binary (yes/no) to "spectral" ("shades of gray"), roughly corresponding to peripheral and central 
TABLE 2. Examples of factors influencing credibility (specific to computer-based media).

\begin{tabular}{l}
\hline \multicolumn{1}{c}{ Source/Medium/Message* } \\
$\begin{array}{l}\text { - Source expertise/knowledge/competence } \\
\text { - Source trustworthiness }\end{array}$ \\
$\begin{array}{l}\text { - Source credentials/influence } \\
\text { - Message content/relevance/currency/accuracy/ } \\
\text { tailoring }\end{array}$ \\
$\begin{array}{l}\text { - Surface attractiveness/format } \\
\text { - Design of interface }\end{array}$ \\
- Speed of loading \\
- Usability/accessibility \\
- Interactivity/flexibility
\end{tabular}

${ }^{a}$ In the literature on credibility of computing technologies, the source and medium are usually treated as the same thing. Many authors also include the message in this category.

routes to persuasion, respectively. However, empirical data does not yet exist to support this model, and neither these authors, nor Petty and colleagues (or in fact others who have examined the issue) are clear on how these assessments occur temporally. For example, are all aspects of the information considered at once for an overall assessment of credibility? Or is there a staged assessment, with information "passed" or "failed" for credibility at each stage? These questions seem especially relevant when the involvement of the medium becomes greater, as is the case for the Internet, and require further study.

Figure 1 presents a possible model of how credibility assessment may occur on-line, based on our interpretation of the available evidence. This is presented as an attempt to synthesize the literature into a framework that will allow for further research to address gaps in what we currently know.

The model is laid out as a staged process with the caveat that this is untested. We propose that the first task for a user is to rate the credibility of the medium itself, based primarily on its surface characteristics. The second task would be rating the source vis-á-vis the content of the message. For both traditional and on-line media, there are fairly consistent markers of source credibility, primarily having to do with trustworthiness and expertise. These can be rated through direct experience with the specific source, through referrals from other credible sources, or through inference based on labels or credentials.

A third aspect of the process would involve assessing the interaction of the message presentation and content with the user's cognitive state. According to persuasion theories, a user who has a high need for the information will overlook weak peripheral cues. If the information-seeking episode is more casual, surface characteristics will have more influence-if they are positive, the user will stay; if not, the user will leave. Thus, if the initial credibility rating is a "fail," then the user will not continue seeking information from

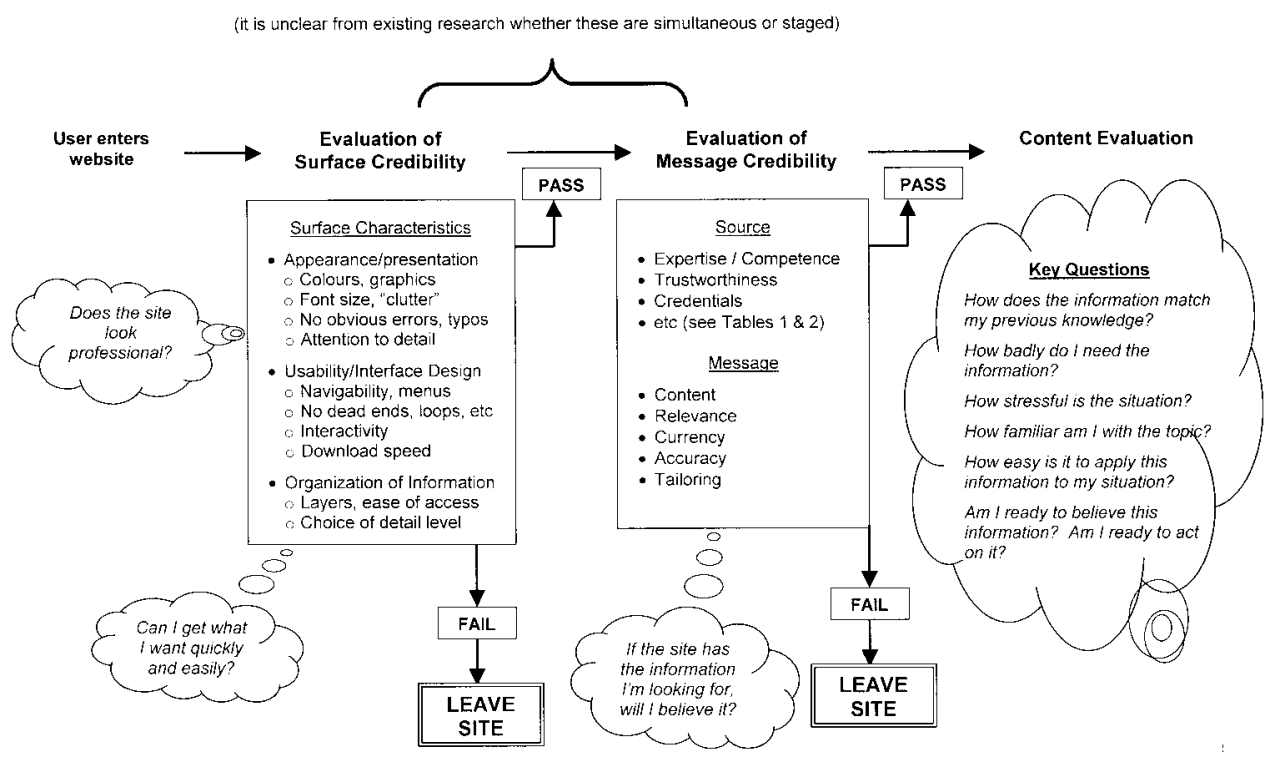

FIG. 1. Proposed model for how users judge the credibility of on-line information. 
that source (Web site). However, if the user is motivated by, for example, stress (Bar-Tal et al., 1999; Burns \& D'Zurilla, 1999; Smith, 1990), and is using the Internet as a helpseeking strategy, or if the user has a high level of personal responsibility in the outcome, finds the information personally relevant or has a high "need for cognition" (Petty et al., 1988), they should persevere beyond the barrier of inconsistent peripheral cues (i.e., bad interface), to the next level of judgment: the information itself. At this point, a favorable credibility perception should lead the user to uptake the information and begin the process of comparing it to currently held knowledge and attitudes to eventually arrive at a decision to be persuaded (or not) and to initiate a behavior change (or not). According to the ELM and other models (e.g., McGuire, 1985), a mitigating factor will be how knowledgeable the user is to begin with. Those already fairly knowledgeable about the topic will tend to scrutinize the information more closely, and more skeptically, with the results often being a decision to reject the information (credibility failure), whether true (incredulity error), or not (correct rejection). Other mitigating factors are time and familiarity.

In summary, our model proposes that the assessment of credibility of Internet information is iterative. Upon entering a Web site, the user makes some immediate judgments about the surface characteristics of the site. The key questions during this assessment are: "Does this site look professional?" and "Can I get what I want quickly and easily?" The appearance, interface design, including download speed and interactivity, and Web site organization are key aspects that the user will evaluate. If this initial evaluation passes the user's criterion, then they will move to the next "level" of evaluation. If the rating is a "fail," the user is likely to leave the site and seek out another. It should be kept in mind that this pass/fail "criterion" would likely differ among users, depending on the contextual factors and intervening variables, such as time, expertise, experience with Web sites/computers, existing knowledge, and need for information, previously described.

The second level of evaluation is of the credibility of the message. The key question at this stage is "If the site has the information I'm looking for, will I believe it?" At this time, aspects of the source and message would be evaluated, as identified in Table 2. Source expertise, competence, trustworthiness, credentials, etc., would be considered, along with the content of the message (including level of detail, examples, alternatives), its accuracy, currency, and relevance to the user need. In addition, the user might evaluate how easy it would be to tailor the information to their own situation. Again, if these assessments meet the user's criteria (caveat as above) for credibility for all (or enough?) of the dimensions, the user accepts the information as credible, and decides to evaluate the information content. If they do not, they will likely leave the site.

The final part of the process is presented for the sake of completeness, with the caveat that a full review of this type of evaluation was beyond the scope of this article. However, the literature reviewed provided some insight to the types of questions that users might consider as they come to evaluate the information itself. The user at this point in their evaluation might ask themselves questions of the following types: (a) How does the information match my previous knowledge? (b) How badly do I need the information? (c) How familiar am I with the topic? (d) How easy is it to apply this information to my situation? (e) Am I ready to believe this information? Am I ready to act on it?

At this point, given the almost limitless number of possible interactions among contextual and intervening variables, the issues become much more complex and difficult to predict. In addition, if the on-line information seeking process, both within and between Web sites, is iterative, the steps themselves are likely not linear, as presented in Figure 1 , but in fact interactive, especially as initial surface and source cues interact. Again, this requires testing.

\section{Conclusions}

So, how do those seeking on-line information decide what to believe? The preceding review shows the multidimensionality of the concept of credibility. A myriad of factors related to source, message, and receiver serve as markers to credibility. The potential interactions of these are seemingly limitless. The relative lack of high quality research evidence leaves many of the key questions unanswered. The emergence of the Internet brings these issues to the forefront, and some key next steps in advancing our understanding of these issues will involve research into the following types of questions: (a) What are the key markers for credibility of on-line information? (b) Are surface characteristics for Web-based media more important than for traditional media? (c) What traditional printed materials provide the best analogy, vis-à-vis "publication authority/ credibility," to Web sites? It might be hypothesized that such items as brochures or pamphlets might be more representative than would books, journal articles, newspapers, etc. (d) Is the model proposed in Figure 1 an accurate representation of how users judge the credibility of Web sites and the information presented on them?

From a more practical perspective, we can start to think about how one should design the "ideal" Web site. Focusing on the key aspects of credibility outlined above, we know that such a site should emphasize a good interface and project a professional image, making use of established design principles, including ease of use, layering, and menus to organize information.

The source credibility of the site should be made obvious by presenting institutional or individual credentials, showing quality ratings awards, and highlighting links to and from other credible Web sites, such as those of respected institutions or consumer advocacy groups (e.g. Mayo Clinic or Cancer Society for health sites). Obtaining a URL suffix denoting educational, nonprofit or government designation can reassure users. Commercial relationships should be avoided, especially those with companies presumed to have 
a vested interest (such as pharmaceutical companies for health Web sites).

The most useful Web sites will be those taking advantage of the interactive properties of the medium (Cassell et al., 1998; McMillan, 1999) and tailored to the beliefs and needs of the audience (Campbell et al., 1999). As with any medium, it is important to present messages clearly and concisely, and judicious use of analogies, examples, and alternatives might also promote proper application of users' cognitive structures, simplifying information processing.

The interactivity of computer applications is constantly evolving. One direction of change is toward increasingly life-like "virtual agents" supporting human-computer interaction that is increasingly similar to human-human interaction. When interactive Web sites include these virtual agents, a new aspect of Web credibility emerges: the same characteristics that mark trusted off-line sources will be used to evaluate these new on-line sources. Human-like virtual agents (Burgoon et al., 2000) and devices (Fogg, 1999) will be able to assume key interpersonal, or "informal" (Olaisen, 1990), credibility markers, such as competence, character, sociability, and influence (Burgoon et al., 2000), leading to a decreased need for Web users to rely on peripheral cues. Key features of "captologically" (Fogg, 1999; see also www.captology.org) successful technologies include the ability to convey credibility, and the use of tried and true strategies from consumer behavior and marketing theory to persuade (King \& Tester, 1999), including: (a) simulating experiences, (b) monitoring or tracking, (c) creating "environments of discovery," (e) motivating virtual groups (i.e., through competition or recognition rewards), and (f) tailoring/personalizing information.

One can begin to imagine how the power of existing, and impending, Internet technologies, especially interactive ones, will change the way that users seek and use on-line information.

\section{References}

Bar-Tal, Y., Raviv, A., \& Spitzer, A. (1999). The need and ability to achieve cognitive structuring: Individual differences that moderate the effect of stress on information processing. Journal of Personality \& Social Psychology, 77, 33-51.

Belkin, N.J. (1978). Information concepts for information science. Journal of Documentation, 34, 55-85.

Buckland, M.K. (1991). Information as thing. Journal of the American Society for Information Science, 42, 351-360.

Bunn, M.D. (1993). Consumer perceptions of medical information sources: an application of multidimensional scaling. Health Marketing Quarterly, 10, 83-104.

Burgoon, J.K., Bonito, J.A., Bengtsson, B., Cederberg, C., Lundeberg, M., \& Allspach, L. (2000). Interactivity in human-computer interaction: A study of credibility, understanding, and influence. Computers in Human Behavior; 16, 553-574.

Burns, L.R. \& D'Zurilla, T.J. (1999). Individual differences in perceived information-processing styles in stress and coping situations: Development and validation of the Perceived Modes of Processing Inventory. Cognitive Therapy \& Research, 23, 345-371.

Campbell, M.K., Bernhardt, J.M., Waldmiller, M., Jackson, B., Potenziani, D., Weathers, B., \& Demissie, S. (1999). Varying the message source in computer-tailored nutrition education. Patient Education \& Counseling, $36,157-169$.

Cassell, M.M., Jackson, C., \& Cheuvront, B. (1998). Health communication on the Internet: An effective channel for health behavior change? Journal of Health Communication, 3, 71-79.

Critchfield, R. (1998). Credibility and web site design. [On-line]. Available: http://www.warner.edu/critchfield/hci/critchfield.html.

Deloitte \& Touche (2000). Health care. 1996-2000. [On-line]. Available: http://www.us.deloitte.com/us/what/indprac/healthcare/healthc.htm.

Dervin, B. (1977). Useful theory for librarianship: Communication not information. Drexel Library Quarterly, 13, 16-32.

Dervin, B. (1992). From the mind's eye of the user: the sense-making qualitative-quantitative methodology. In J.D.Glazier \& R.R.Powell (Eds.), Qualitative research in information management (pp. 61-84). Englewood, CO: Libraries Unlimited.

Dervin, B. (1997). Given a context by any other name: Methodological tools for taming the unruly beast. In P. Vakkari, R. Savolainen, \& B. Dervin (Eds.), Information seeking in context. London: Taylor Graham.

DMOZ Open Directory Project. Health. [On-line]. Available: http://www. dmoz.org/Health/.

e-land. (2000). EAmericas Report: Sneak Preview. [On-line]. Available: http://www.emarketer.com/estats/s_eamr_prev.html.

Eysenbach, G. (2000). Consumer health informatics. BMJ, 320, 17131716.

Eysenbach, G., \& Diepgen, T.L. (1998). Towards quality management of medical information on the Internet: Evaluation, labelling, and filtering of information. BMJ, 317, 1496-1502.

Feightner, J.W., Quintana, Y., Marshall, J.N., \& Wathen, C.N. (1999). Electronic dissemination of Canadian clinical practice guidelines to health care professionals and the public. Ottawa, Health Canada. Available: http://www.hc-sc.gc.ca/hppb/healthcare/pubs/edccpg/index.html.

Fogg, B.J. (1999). Persuasive technologies-Now is your chance to decide what they will persuade us to do-and how they'll do it. Communications of the ACM., 42, 26-29.

Fogg, B.J., \&. Tseng, H. (1999). The elements of computer credibility. Paper presented at the Conference on Human Factors and Computing Systems, Pittsburgh, PA, May 15-20, 1999.

Fogg, B.J., Marshall, J., Laraki, O., Osipovich, A., Varma, C, Fang, N., Paul, V., Rangnekar, A., Shon, J., Swani, P., \& Treinen, M. (2000). Elements that affect Web credibility: Early results from a self-report study. [presentation online]. Available from URL: (accessed 29 September 2000).

Hovland, C.I., Janis, I.L., \& Kelley, H.H. (1953). Communication and persuasion. New Haven, CT: Yale University Press.

Hovland, C.I., Lumsdaine, A.A., \& Sheffield, F.D. (1949). Experiments on mass communication. Princeton, NJ: Princeton University Press.

Impicciatore, P., Pandolfini, C., Casella, N., \& Bonati, M. (1997). Reliability of health information for the public on the World Wide Web: Systematic survey of advice on managing fever in children at home. BMJ, 314, 1875-1879.

Jadad, A.R., \& Gagliardi, A. (1998). Rating health information on the Internet: Navigating to knowledge or to Babel? JAMA, 279, 611-614.

Jadad, A.R., Haynes, R.B., Hunt, D., \& Browman, G.P. (2000). The Internet and evidence-based decision-making: A needed synergy for efficient knowledge management in health care. CMAJ, 162, 362-365.

Johnson, T.J., \&. Kaye, B.K. (1998). Cruising is believing?: Comparing Internet and traditional sources on media credibility measures. Journalism and Mass Communication Quarterly, 75, 325-340.

Kim, P., Eng, T.R., Deering, M.J., \& Maxfield, A. (1999). Published criteria for evaluating health related web sites: Review. BMJ, 318, 647-649.

King, P., \& Tester, J. (1999). The landscape of persuasive technologies. Communications of the ACM, 42, 31-44.

McClung, H.J., Murray, R.D., \& Heitlinger, L.A. (1998). The Internet as a source for current patient information. Pediatrics, 101(6), E2.

McGuire, W.J. (1985). Attitudes and attitude change. In G. Lindzey \& E. Aronson (Eds.), The handbook of social psychology (3rd ed., pp. 233346). New York: Random House. 
McMillan, S.J. (1999). Health communication and the Internet: Relations between interactive characteristics of the medium and site creators, content, and purpose. Health Communication, 11, 375-390.

Nass, C., \& Moon, Y. (2000). Machines and mindlessness: Social responses to computers. Journal of Social Issues, 56(1), 81-103.

O'Hara, B., Netemeyer, R., \& Burton, S. (1991). An examination of the relative effects of source expertise, trustworthiness, and likability. Social Behavior \& Personality, 19, 305-314.

Olaisen, J. (1990). Information quality factors and the cognitive authority of electronic information. In I. Wormell (Ed.), Information quality: Definitions and dimensions (pp. 91-121). London: Taylor Graham.

Petty, R.E., \& Cacioppo, J.T. (1986). Elaboration likelihood model. In L. Berkowitz (Ed.), Advances in experimental social psychology (vol. 19, pp. 123-205). San Diego, CA: Academic Press.

Petty, R., \& Cacioppo, J. (1990). Involvement and persuasion: Tradition versus integration. Psychological Bulletin, 107, 367-374.

Petty, R., Cacioppo, J., Sedikides, C., \& Strathman, A. (1988). Affect and persuasion: A contemporary perspective. American Behavioral Scientist, 31, 355-371.

Quintana, Y., Feightner, J.W., Wathen, C.N., Sangster L.M., \& Marshall, J.N. (2001). Preventative health information on the Internet. Qualitative study of consumers' perspectives. Canadian Family Physician, 47, 1759-1765.

Richards, B., Colman, A.W., \& Hollingsworth, R.A. (1998). The current and future role of the Internet in patient education. International Journal of Medical Informatics. 50, 279-285.

Rieh, S.Y., \& Belkin, N.J. (1998). Understanding judgment of information quality and cognitive authority in the WWW. In C.M. Preston (Ed.), Proceedings of the 61st ASIS Annual Meeting (pp. 279-289). Silver Spring, MD: American Society for Information Science.

Reih, S.Y., \& Belkin, N.J. (2000) Interaction on the Web: Scholars' judgment of information quality and cognitive authority. In D.H. Kraft
(Ed.), Proceedings of the 63rd ASIS Annual Meeting (pp. 25-33). Silver Spring, MD: American Society for Information Science.

Robinson, T.N., Patrick, K., Eng, T.R., \& Gustafson, D. (1998). An evidence-based approach to interactive health communication: A challenge to medicine in the information age. Science Panel on Interactive Communication and Health. JAMA, 280, 1264-1269.

Rogers, E.M. (1995). Diffusion of Innovations (4th ed.). New York: The Free Press.

Self, C.S. (1996). Credibility. In M. Salwen \& D. Stacks (Eds.), An integrated approach to communication theory and research. Mahwah, NJ: Erlbaum.

Shon, J. \& Musen, M.A. (1999). The low availability of metadata elements for evaluating the quality of medical information on the World Wide Web. Proc. AMIA Symp.(pp. 945-949).

Silberg, W.M., Lundberg, G.D., \& Musacchio, R.A. (1997). Assessing, controlling, and assuring the quality of medical information on the Internet. JAMA, 277, 1244-1245.

Slater, M.D. \& Rouner, D. (1996). How message evaluation and source attributes may influence credibility assessment and belief change. Journalism and Mass Communication Quarterly, 73, 974-991.

Smith, A. (1990). Stress and information processing. In M.Johnston \& L. Wallace (Eds.), Stress and medical procedures (pp. 58-79). Oxford: Oxford University Press.

Tseng, S. \& Fogg, B.J. (1999). Credibility and computing technology. Communications of the ACM, 42, 39-44.

Wilson, E., \& Sherrell, D. (1993). Source effects in communication and persuasion research: A meta-analysis of effect size. Journal of the Academy of Marketing Science, 21, 101-112.

Winker, M.A., Flanagin, A., Chi-Lum, B., White, J., Andrews, K., Kennett, R.L., DeAngelis, C.D., \& Musacchio, R.A. (2000). Guidelines for medical and health information sites on the Internet: Principles governing AMA web sites. JAMA, 283, 1600-1606. 